\title{
BIOÉTICA EN NICARAGUA
}

\author{
Armando Ulloa González* y Melba de la Cruz Barrantes Monge**
}

Resumen: Este trabajo describe la situación de la bioética en Nicaragua, caracterizando las circunstancias y el contexto de las actividades de educación médica y las unidades prestadoras de servicios de salud.

El desarrollo de un nuevo modelo de atención integral en salud, la implementación de políticas de salud que garanticen a la población el mayor acceso y gratuidad a los servicios, y los cambios acontecidos en los cuidados médicos, debidos en parte al reconocimiento creciente de una mayor autonomía de los pacientes y al uso creciente de nuevas tecnologías médicas, hace que se presenten algunas limitantes y dilemas en las unidades asistenciales y entre el personal de salud.

La bioética en Nicaragua tiene un desarrollo incipiente: no está institucionalizada ni se han previsto los mecanismos formales que permitan resolver los problemas éticamente complejos, por lo tanto, constituye un gran reto por parte de las instituciones educativas y rectoras de la salud.

Palabras clave: bioética, instituciones de salud, instituciones educativas, Nicaragua

\section{BIOETHICS IN NICARAGUA}

\begin{abstract}
This work describes the bioethics situation in Nicaragua, with information on the circumstances and the context of activities in medical education and health care providers.

The development of a new model of comprehensive health care-the implementation of health care policies that guarantee Nicaraguans better access to care and services free of charge, and the changes that have occurred in medical care, due in part to the growing recognition of greater patient autonomy and the increase in the use of new medical technologies-all have contributed in some part to current obstacles and dilemmas in medical clinics and between health care personnel.

Bioethics in Nicaragua is in the initial stage of development. It is neither institutionalized nor is it subject to formal mechanisms that permit the resolution of complex ethical problems, thus presenting an important challenge for institutions of higher learning and decision-makers in health.
\end{abstract}

Key words: bioethics, health care institutions, educational institutions, Nicaragua

\section{BIOÉTICA EM NICARÁGUA}

Resumo: Este trabalho descreve a situação da bioética na Nicarágua, caracterizando as circunstâncias e o contexto das atividades de educaçấo médica e as unidades prestadoras de serviços de saúde.

O desenvolvimento de um novo modelo de atenção integral em saúde, a implementação de políticas de saúde que garantam à população maior acesso e gratuidade aos serviços, e as mudanças ocorridas nos cuidados médicos, devidas em parte ao reconhecimento crescente de uma maior autonomia dos pacientes e ao uso crescente de novas tecnologias médicas, determinam que se apresentem alguns dilemas e limitantes nas unidades assistenciais e entre o pessoal de saúde.

A bioética na Nicarágua tem um desenvolvimento incipiente: não está institucionalizada nem se acham previstos os mecanismos formais que permitam resolver os problemas eticamente complexos, portanto, constitui um grande desafio por parte das instituiçốes educativas e gestoras da saúde.

Palavras-chave: bioética, instituiçôes de saúde, instituições educativas, Nicarágua

\footnotetext{
* Doctor en Medicina y Cirugía. Vicedecano de la Facultad de Medicina, Universidad Nacional Autónoma de Nicaragua, Managua, Nicaragua Correspondencia: aulloagonzal@yahoo.com

** Doctora en Medicina y Cirugía. Oficina de Investigación e Información Médica de la Dirección de Regulación Sanitaria, Ministerio de Salud, Nicaragua
} 


\section{Introducción}

Nicaragua ocupa el segundo lugar entre los países más pobres y endeudados de América Latina, y se ubica en el grupo de desarrollo humano medio. Esto ha generado inequidad, injusticia y una brecha importante para alcanzar un adecuado estado de bienestar y de salud de la población.

Durante las últimas dos décadas el país ha transitado por un intenso proceso de ajuste estructural, reforma del Estado y problemas de gobernabilidad, derivados de la situación económica y política, lo que ha ocasionado inestabilidad que impacta negativamente en la calidad de vida. Según datos de la Organización Panamericana de la Salud (OPS), 45\% de la población en Nicaragua vive en condiciones de pobreza, hay inequidad en el financiamiento de la salud y entre 35 y $40 \%$ de la población no tiene acceso a la salud.

Por otra parte, el Sistema de Salud tiene un presupuesto deficitario y poca capacidad de respuesta ante las demandas cada vez más crecientes; la relación médico paciente tiene un predominio paternalista, lo que conlleva que nuestros profesionales se vean inmersos en una serie de dilemas éticos.

No obstante la conformación de comités y la incorporación de la enseñanza de la bioética, Nicaragua todavía presenta algunas limitantes para abordar sistemáticamente esta dimensión, debido a la escasez de recursos humanos formados y a la exigua implementación de instancias que ayuden a resolver dilemas.

Los nuevos desafíos han llevado a la búsqueda de procedimientos que garanticen la educación y un proceder profesional que vele por el respeto al ser humano, de manera que preservar la dignidad humana sea el fin de la toma de decisión.

\section{Antecedentes de la bioética en Nicaragua}

La bioética en Nicaragua no está institucionalizada. Ciertas iniciativas han surgido de intereses locales, por lo que, desde un punto de vista orgánico y formal, tiene un desarrollo incipiente. Hace un poco más de una década surge en las instituciones universitarias, ante la necesidad de velar por el respeto a la dignidad y los derechos del ser humano que participa en investigaciones. Hay cierta experiencia en algunos hospitales al crearse comités cuyo propósito es cumplir los preceptos médicos y las normas éticas en la atención médica.

Sin embargo, aparecen también ciertos antecedentes en formación en ética médica, en la cual se establecen normas para el ejercicio de la profesión y la necesidad de incorporar contenidos en los planes de estudios universitarios. Debido a que el desarrollo de la ética médica y la bioética en Nicaragua ha tenido mucha relación con la enseńanza de la medicina, se abordará una breve reseńa de ambos aspectos y el vínculo existente.

La enseñanza de la medicina en Nicaragua tiene un poco más de dos siglos pues, según Carlos Tünnerman, la primera cátedra de medicina fue fundada en 1798 por el obispo José Antonio de la Huerta. Durante esa época el país era una colonia dependiente de las influencias de España y la educación se desarrollaba bajo ese influjo.

Los primeros médicos se formaban en conventos y monasterios donde se empapaban de las viejas concepciones escolásticas, el protomedicato se encargaba de la formación médica, del ejercicio de la profesión y de la aplicación de normas éticas.

Uriel Guevara narra que, al fundarse la universidad en 1812, la cátedra de Teología y Derecho Canónico regulaba aspectos éticos. Posteriormente, al aprobarse la Facultad de Ciencias Médicas en 1887, se creó la cátedra de Historia de la Medicina, Ética y Deontología Médica, la cual fue abolida en 1960 por reducciones del currículo médico.

Con la llegada del siglo XIX -que significó no sólo la libertad sino también cambios educativos, ya que en las últimas décadas la medicina adoptó un carácter científico y se introdujo la medicina clínica-, en León, la ciudad universitaria, se instaura y se oficializa la profesión médica al organizar académicamente la Facultad que en 1893 empieza a otorgar los primeros títulos de "doctor en Medicina".

En ese período se asiste a una medicina de carácter técnico pero que mantiene una influencia religiosa, predominante en esa época. Se enseña una concepción de la práctica médica paternalista y de carácter religioso, en la que se procura el bien de los semejantes como un imperativo benevolente y de caridad. A los cuidados del enfermo se unían la oración y la unción 
sacramental como un elemento común de la práctica médica.

Un poco avanzado el siglo XX la formación médica recibe una gran influencia de la medicina francesa, desarrollando una práctica basada en la clínica y con una tendencia hacia la secularización, lo que implicó un cambio de valores y conductas. El carácter técnico científico de la medicina da una mayor confianza a los médicos para una actuación con mayor independencia, ya que, cada vez más, se va haciendo posible la curación de las enfermedades y la certeza más creciente de su evitabilidad.

A lo largo de ese siglo Nicaragua se vio envuelta en diversos conflictos y luchas sociales internas y externas, como consecuencia de la injusticia social que provocaron los diferentes gobiernos al no garantizar derechos humanos esenciales para la vida y dignidad humana, como el derecho a la salud, la educación, el trabajo y una vivienda digna.

A los factores mencionados se suma el proceso de ajuste estructural iniciado en 1990, las reformas del Estado y los problemas de gobernabilidad derivados de la situación económica y política que impactan negativamente en la calidad de vida y en la poca capacidad de respuesta del sistema de salud.

Un hecho relevante fue la creación del Sistema Nacional Único de Salud, en 1979, como una de las políticas impulsada por la Revolución Popular Sandinista. Se cambió el modelo concentrado en la atención hospitalaria hacia un modelo de Atención Primaria de Salud. Se establece como principio fundamental que la salud es un derecho de todos los individuos y una responsabilidad del Estado, garantizando que los servicios sean integrales y accesibles a toda la población.

A pesar de lo anterior, la distribución de recursos poco equitativa ha sido una constante a lo largo de la historia del país, ello debido a que no ha sido abordada desde un enfoque ético, siendo el sustrato esencial la poca correspondencia entre la respuesta moral y ética y el ejercicio del poder.

\section{Situación actual de la bioética}

\section{Bioética en instituciones educativas de Nicaragua}

El desarrollo incipiente de la bioética en Nicaragua se evidencia en que sólo se ha documentado la existencia de dos comités de ética para investigaciones biomédicas, impulsados por las facultades de Ciencias Médicas de las universidades Nacional Autónoma de Nicaragua - de la ciudad de León- y de Managua.

Por ser la universidad una institución académica, vinculada con el desarrollo de las investigaciones biomédicas, representa un espacio propicio para la reflexión y análisis de las preocupaciones bioéticas. En la Facultad de Ciencias Médicas de la Universidad Nacional Autónoma de Nicaragua (UNAN-León) se creó, en abril del 1995, el primer Comité de Ética para Investigaciones Biomédicas (CEIB). En su par de Managua, en cambio, la creación del Comité fue sólo en 2006, para evaluar estudios de investigación farmacológicos. Conformado por cuatro integrantes permanentes, está en proceso de formalizar los procedimientos y mecanismos de constitución y funcionamiento.

El CEIB de la Facultad de Ciencias Médicas de León ha venido desarrollando acciones en el campo de la bioética en investigaciones biomédicas a lo largo de los últimos diez años. Surge ante el imperativo creciente de evaluar las investigaciones, ampliar la colaboración y realizar convenios con diversos organismos nacionales e internacionales.

La creación de este comité fue posible por la intervención de Uriel Guevara Guerrero, maestro de muchas generaciones de médicos, con una dilatada experiencia profesional que conjugaba lo científico y humanístico en su trabajo docente.

El CEIB UNAN-León constituye el primer organismo rector oficial en los aspectos éticos de las investigaciones biomédicas del país. Está integrado por académicos de la comunidad científica, médicos y profesionales de la salud no médicos, trabajadores sociales, abogados, ministros religiosos, administrativos, representantes de la comunidad y de los derechos humanos, o del Ministerio de la Familia y la Niñez, y es coordinado por el vicedecanato de la facultad. En 2003 el CEIB fue acreditado por el Departamento de Salud y Servicio de Protección Humana de los Estados Unidos.

Es el único comité que cuenta con normas para el funcionamiento y guías para la realización de las evaluaciones. También establece los procedimientos y mecanismos para la selección y requisitos de los miembros. El comité ha elaborado guías operativas para revisión de tesis, procedimientos de intervención, supervisión y seguimiento. 
La Facultad de Ciencias Médicas de UNAN-León trabaja contenidos sobre aspectos de la bioética para la formación médica de manera transversal, en los cuales se abordan diversos tópicos: el error médico, ética en investigaciones, la relación médico-paciente, el secreto profesional, la colegiación en especialidades médicas y quirúrgicas, entre otros. Asimismo, ha desarrollado talleres y cada año organiza un simposio, lo que permite un espacio para discutir temas relevantes.

Las facultades de medicina de universidades privadas no cuentan con comités de bioética y aquellas facultades que realizan investigaciones de carácter académico solicitan la aprobación del trabajo en la institución en la cual se realizará el estudio. La Universidad Americana (UAM) tiene un instituto que tiene como eje los aspectos éticos del desarrollo humano. También incorpora la enseñanza de la bioética en dos asignaturas: la ética médica orientada a los aspectos éticos de la práctica médica y otro programa denominado "bioética”, en el cual desarrolla principios éticos aplicados a la práctica profesional e investigativa, bioética en grupos vulnerables e incompetentes, y aspectos legales.

\section{Bioética en instituciones prestadora de salud del sector público}

En el ámbito de la atención en salud no se han conformado comités de ética en las instituciones. No obstante, ciertas iniciativas van encaminadas a desarrollar su aplicación, entre ellas se destaca la elaboración de formularios de consentimiento informado en el Hospital Alemán Nicaragüense y la existencia de un Código de Ética en el Hospital Infantil Manuel de Jesús Rivera, aprobado por la dirección del hospital.

En agosto de 2007, por iniciativa de la Dirección de Regulación Sanitaria y de la Dirección de Docencia e Investigación del Ministerio de Salud, se conformó un comité ad hoc del Ministerio para las evaluaciones de los protocolos de investigación de instituciones extranjeras y casas farmacéuticas.

De acuerdo con la encuesta realizada por Armando Ulloa y Melba Barrantes, aplicada en ocho Sistemas Locales de Atención Integral en Salud (SILAIS) del país y cinco centros hospitalarios de Managua, con el fin de conocer la situación de la bioética en las instituciones de salud del sector público, se encontró lo siguiente:

\begin{tabular}{|l|l|}
\hline - Comités de bioética & No existen conformados oficialmente para investigaciones y de atención hospitalarios. \\
\hline $\begin{array}{l}\text { - Dictamen sobre } \\
\text { propuestas de investigación }\end{array}$ & $\begin{array}{l}\text { 63\% refirió que conforman comité ad hoc para evaluar propuestas de investigación específicas, en el cual } \\
\text { se integran autoridades hospitalarias y académicas. 10\% indicó que son evaluados por otras instituciones } \\
\text { (extranjeras y/o nacionales), entre ellas el Comité de Investigación de la UNAN-León. Otro 10\% señaló } \\
\text { que las decisiones son tomadas por las autoridades superiores y ambos a la vez. }\end{array}$ \\
\hline $\begin{array}{l}\text { - Solución de problemas } \\
\text { para la atención médica }\end{array}$ & $\begin{array}{l}50 \% \text { declaró que es a través de un comité ad hoc; 25\% que es decidido por el paciente y 8\% que es decidido } \\
\text { por el médico tratante. }\end{array}$ \\
\hline $\begin{array}{l}\text { - Integrantes de estos } \\
\text { comités para solución de } \\
\text { dilemas en la atención } \\
\text { médica }\end{array}$ & $\begin{array}{l}\text { Generalmente está integrado por las autoridades hospitalarias y jefes de servicios. El comité del Centro } \\
\text { Nacional de Radioterapia, conformado por un equipo multidisciplinario (médico tratante, psicólogos y } \\
\text { trabajadores sociales, entre otros), ha implementado el consentimiento informado. En el Centro Nacional de } \\
\text { Cardiología participan profesionales propios de la especialidad que evalúan las solicitudes de procedimientos } \\
\text { hemodinámicos. }\end{array}$ \\
\hline - Códigos de ética & $\begin{array}{l}\text { El Hospital Infantil Manuel de Jesús Rivera, "La Mascota", es el único centro asistencial con un código de } \\
\text { ética elaborado por el comité del hospital con el propósito de dar cumplimiento a los preceptos médicos } \\
\text { más importantes. Se incluyen allí los deberes de los médicos para con sus pacientes, con sus colegas, con } \\
\text { la institución donde se labora, con los profesionales afines, con las juntas médicas y con las investigaciones } \\
\text { intrahospitalarias. }\end{array}$ \\
\hline $\begin{array}{l}\text { - Enseńanza de la bioética } \\
\text { en las unidades de salud }\end{array}$ & $\begin{array}{l}\text { 23\% señala que lo ha previsto; 38\% expresó que han realizado actividades relacionadas, como talleres y debates, } \\
\text { siendo los temas más relevantes el aborto terapéutico, la relación médico-paciente e inequidad en salud. }\end{array}$ \\
\hline
\end{tabular}

\section{Aspectos legales}

En los últimos años, Nicaragua ha avanzado en establecer un marco legal para la regulación de las investigaciones en salud. Ejemplo de ello es la Ley General de Salud (423), la cual señala que son competencias y atribuciones del Ministerio de Salud regular y promover la investigación científica en salud, biomédica y el desarrollo de la transferencia tecnológica dentro del sector. Asimismo, establece que las investigaciones deben regirse por los principios científicos y éticos 
internacionalmente aprobados, y la necesidad de contar con el consentimiento informado para la realización de investigaciones. En la reglamentación de dicha ley se precisa el objetivo y funcionamiento del Tribunal de Bioética para las investigaciones.

En 2003 se estableció el Comité Nacional de Investigaciones en Salud (CONIS), mediante acuerdo ministerial, formulando su reglamentación, estableciendo las funciones de los comités éticos de investigación científica, de acuerdo con la Declaración de Helsinki, las normas CIOMS y otras pautas éticas internacionales.

Otra ley que promoverá la bioética en el país es la creada por el Colegio de Médicos y Cirujanos de Nicaragua, presentada el 8 de septiembre de 1995 por el gremio ante la Asamblea Nacional, en la cual se destaca que: "El Colegio Médico tiene como fines, como organismo único, integrador y regulador del ejercicio de la profesión médica, en lo ético y deontológico, vigilar que el ejercicio de esta profesión cumpla la función social". Uno de los objetivos es: "Desarrollar el código de ética que regule el ejercicio de la profesión médica velando por el prestigio y dignidad de la misma, así como de mantener incólume la integridad moral, científica y profesional de los médicos, en beneficio de los pacientes". Entre sus funciones está la de velar por la honestidad, la eficiencia técnica y el mantenimiento de la ética en el ejercicio de la profesión médica, reglamentar y vigilar que se cumplan los aspectos legales y bioéticos de la profesión, y establecer, aplicar y propiciar reformas al Código de Ética Médica.

\section{Temas de bioética de interés nacional}

En la última década el país ha sido también escenario de una discusión pública sobre temas relevantes, como el aborto terapéutico, la contracepción, el uso del preservativo para prevenir el contagio por el VIH/SIDA y la justicia en los cuidados públicos.

La Asamblea Nacional de Nicaragua aprobó el 27 de octubre de 2007 la Ley 603 que prohíbe el aborto terapéutico en Nicaragua, reconocido en 1881 en el artículo 165 del Código Penal Nicaragüense, el cual señalaba que: "el aborto terapéutico será determinado científicamente con la intervención de tres facultativos, por lo menos, y el consentimiento del cónyuge o pariente de la mujer para los fines legales". Esta derogación no permite ninguna excepción, ni para embarazos por violación o incesto que se contemplaban anteriormente, lo que plantea un grave dilema para profesionales y pacientes. Una de las objeciones a la prohibición es que provocará que muchas mujeres mueran, ya que la ley no protegería sus vidas en caso de embarazos de riesgo.

Otro tema que reviste importancia en la actualidad y que ha sido planteado por diversos sectores de la sociedad, en particular entre el gremio médico, son las actuaciones reñidas con la ética de los profesionales de la salud, como la mala práctica, el alto costo de los fármacos, automedicación, salario, honorarios de la consulta privada, situación de atención hospitalaria, entre otros.

\section{Conclusiones}

La bioética en Nicaragua tiene un desarrollo incipiente y no está actualmente institucionalizada. Ciertas experiencias de atención hospitalaria y evaluación de investigaciones biomédicas, así como la incorporación de la disciplina en el currículo médico, se han venido suscitando de manera creciente. No obstante, se plantean enormes retos para mejorar la calidad de atención médica y promover los derechos de las personas, ya sea incidiendo en la organización de comités de ética institucional, impulsando normativa jurídica, formando recursos humanos en bioética e incidiendo en las políticas públicas y legislación.

Para alcanzar este propósito se hace conveniente propiciar la conformación del Comité Nacional de Bioética, una regulación legal básica y políticas sanitarias con contenido ético.

Es de alta prioridad contar con una reglamentación que defina la normativa de funcionamiento y evaluación de los comités de ética. Se deben consolidar estos comités en investigación, en las diversas instancias de formación y en los servicios de salud. Asimismo, establecer pautas para conformar los hospitalarios. Todo esto implica efectuar un proceso de capacitación a profesionales de salud e incorporar contenidos de bioética al currículo de grado y posgrado. 


\section{Bibliografía}

Ayón A. Código de Ética en el Hospital del Niño. La Prensa 2002; abril 09, Sección Opinión.

Espinosa Ferrando J. Nicaragua: El desarrollo del Sistema de Salud: Desde mediados del siglo XX hasta nuestros dias. Managua: Friedrich Ebert Stiftung; 2003.

González JA. Interpretar y deconstruir el sentido y la acción política. En Lisón C. Antropología: Horizontes interpretativos. Granada: Universidad de Granada; 1969.

Guevara U, Pacheco Solís N. Manual de Bioética. León: Editorial Universitaria; 2005.

Asamblea Nacional. Ley General de Salud (423). La Gaceta 2002; mayo, Managua, Nicaragua.

Organización Panamericana de la Salud. Derogación del aborto terapéutico en Nicaragua: Impacto en salud. Managua, Nicaragua: OPS; 2007.

Pérez G, Ulloa A, Ramos D. Recordando con el corazón... Construimos el futuro. Veintitrés años de historia de la Facultad de Medicina. Managua: UNAN-Managua; 2003.

Propuesta de Ley creadora del Colegio de Médicos y Cirujanos de Nicaragua. Paginas Verdes 1999; 121. Disponible en: www. euram.com.ni/pverdes/articulos/Ley_de_colegio_medicos_cirujanos_ed121.htm

Recibido: 13 de marzo de 2008

Aceptado: 15 de abril de 2008 